a sodium chloride-like structure. Layers of TIX parallel to the $a b$ plane are spaced out at distances depending on the length of the alkyl chains, which are attached to the thallium ions perpendicularly to these layers. The $a$ and $b$ cell dimensions are almost independent of the alkyl group and vary between $4 \cdot 29 \mathrm{~A}$. (chloride) and $4 \cdot 76 \mathrm{~A}$. (iodide). These spacings, which, except in the case of the iodide, are much lower than the values $4.85 \mathrm{~A}$. (Müller) ${ }^{1}$ and $4.76 \mathrm{~A}$. (Bernal) ${ }^{2}$ found for the distance between rotating chains, are in agreement with other indications that the chains in these compounds do not rotate.

There is evidence that the chains are not of the usual zigzag pattern, but are like Müller and Shearer's ${ }^{3}$ suggested type 3 , and the two covalencies of the central thallium atom in the chain are at $180^{\circ}$.

We have also studied the double refraction of these compounds, which combine the characteristics of both chain and layer structures. The following table shows that the sign of the double refraction may be either positive or negative according as the influence of the chain or layer character is the greater (cf. Bernal's observation, loc. cit., of negative double refraction in an alkyl ammonium chloride).

\section{Sign of Double Refraction}

\begin{tabular}{|c|c|c|c|}
\hline $\mathrm{X}$ & $\mathrm{TiMe}_{2} \mathrm{X}$ & $\mathrm{TIEt}_{2} \mathrm{X}$ & $\operatorname{TIPr}_{2} \mathrm{X}$ \\
\hline $\mathrm{I}$ & - & - & - \\
$\mathrm{Br}$ & - & + & - \\
$\mathrm{CI}$ & + & + & + \\
\hline
\end{tabular}

The variations can be satisfactorily explained by a simple extension of the method of Wooster, ${ }^{*}$ by taking into account the relative importance of the various atoms in their contribution to the refraction.

A detailed account is in preparation.

H. M. Powell.

D. M. Crowfoot

Department of Mineralogy,

University Museum, Oxford.

NATURE, 129, 436, March 19, 1932

2 NATURE, 129, 870, June 11, 1932

J.C.S., 123, $3156 ; 1923$.

Z. Krist., 80, $495 ; 1931$

\section{A Metagalactic Gloud between Perseus and Pegasus}

DURING the course of an investigation concerning the occurrence of bright metagalactic clusters, I have found between the constellations Perseus and Pegasus a number of clusters of anagalactic nebulæ which seem to form subsystems of an extended metagalactic cloud. This cosmical unit can be traced in Herschel's G.C. and is very conspicuous in the N.G.C. and I.C. material. On the whole, the supersystem is composed of fifteen or nineteen larger and smaller metagalactic clusters, situated between $\alpha=23^{\mathrm{h}}$, $\delta=+20^{\circ}$ and $\alpha=3^{\mathrm{h}} 20^{\mathrm{m}}, \delta=+48^{\circ}$. All the subsystems are arranged in a zone reminding one of a region of the galaxy. The well-known Perseus cluster, discovered by M. Wolf, is involved on the north-following boundary, and the Pisces group (E. P. Hubble) near the central region of the cloud. A connexion between the metagalactic cloud in question and the Pegasus cluster is suggested.

450 N.G.C. objects are situated in the general field of the cloud, and the main bulk of these objects seem to be physical members of the cloud. The density of nebulæ in this part of the sky is remarkable. Thus we count 117 N.G.C. objects in the central region, but only 8 and 3 respectively in equal large areas, situated north and south of the said region. Translating the literal symbols of magnitudes given in N.G.C. into numerical values of total magnitudes, we obtain for 312 nebulæ, obviously belonging to the cloud, a frequency - curve of total magnitudes remarkably similar to the corresponding curve for the objects in the bright Virgo cloud, discovered and studied by H. Shapley. Thus the curve also shows the " hump' in its ascending branch, earlier found by W. Baade in the case of the Ursa Major cluster.

The distance of the bright metagalactic supercluster (or cloud of galaxies) can be assigned the preliminary value of $10 \times 10^{6}$ light-years, the extension and depth of the cloud to about $30 \times 10^{6}$ light-years in accordance with the distances found for the known clusters of faint nebulæ involved in the metagalactic cloud here considered.

Observatory, Lund, April 25.

\section{Energy of Dissociation of Nitrogen}

IN Nature of June 11, page 870, A. K. Datta gives the energy of dissociation of nitrogen as deduced from his measurements of the absorption spectrum of nitrous oxide.

The value - 18.1 appears to have been used for the heat of formation of the latter gas. This value is so suspect that a redetermination has been made in this department, ${ }^{1}$ and similar work is in progress at the National Physical Laboratory. ${ }^{2}$ Using the value ${ }^{1}$ $-20 \cdot 6$, Datta's figure becomes $9 \cdot 0_{0}$ volts or 208 kilocalories.

The following table includes the more recent values obtained by various methods, and shows that the energy of dissociation of nitrogen is now comparatively well known:

\begin{tabular}{|l|l|l|}
\hline Birge . & $9 \cdot 04 \pm 0 \cdot 2$ volts & $\begin{array}{l}\text { NATURE, 122, 842; } \\
1928 \\
\text { T.Faraday Soc., 25, } \\
713 ; 1929 \\
\text { Proc. Nat. Acad., 15, } \\
226 ; 1929 \\
\text { Phys. Rev., 39, 224; } \\
1932 \\
\text { KaTURE, 129, 870; } \\
1932\end{array}$ \\
\hline Tate and Lozier & $\begin{array}{l}9 \cdot 4 \pm 0.5 \text { volts } \\
\text { Datta (corrected) }\end{array}$ & $9 \cdot 0$ volts \\
\hline
\end{tabular}

Research Department,

T. C. SUTTON. Woolwich,

June 12.

I T. C. Sutton, Phil. Mag., 1932 (in press).

Annual Reports, 1931, 13 and 75.

\section{Fatuoids or False Wild Oats}

Mr. E. T. Jones's observations ${ }^{1}$ are, as he remarks, highly important in their bearing on the problem of the origin of fatuoids or false wild oats. The conclusions to be drawn from these observations are, however, in my opinion, precisely opposite to those drawn by Mr. Jones. His data, showing that two or more genes are concerned in the production of the fatuoid complex in diploid $\times$ tetraploid oat hybrids surely support my assumption that the fatuoid com. plex of hexaploids is determined by a number of linked genes. Therefore, for fatuoids to arise by gene mutation, as he considers they do, it is necessary to assume mutation occurring simultaneously in all these genes-a rather improbable assumption. The change in dominance, stressed by Mr. Jones, has in other cases been shown to be directly attributable to polyploidy.

As for the absence of crossing-over in fatuoids, this is a characteristic of chromosome aberrations in

No. 3273, VoL. 130] 